\title{
Assembly Path Planning based on Virtools
}

\author{
Yonggang Bai ${ }^{1, a^{*}}$, Fayuan $\mathrm{Wei}^{1, \mathrm{~b}}$ \\ ${ }^{1}$ Institute of Systems Engineering, China Academy of Engineering Physics, Sichuan,China \\ a411baiyg@caep.cn, bweify@caep.cn
}

Keywords: Assembly Path Planning, Interactive Planning, Ant Colony Algorithm, Space Depth Map

\begin{abstract}
On the basis of an established product assembly sequence, Assembly path planning is used to find a track for the part moving from the storage position to the assembly location. On one hand, Path planning can meet the needs of operation and avoid the obstacles, at the same time regarding security as the main purpose, on the other hand, it was an important means of verifying product design and assembly sequence planning.

Considering the use of ant colony Algorithm in the two-dimensional path planning, the assembly space depth map was introduced into 3D assembly path planning with the ant Colony algorithm ,which was based on depth-first search, for exploring the assembly path of the components. With the help of Virtools, which could provide 3D virtual environment and real-time collision detection, validate the results of the preliminary plan, and improve the planned path through interactive. It's possible, through the integration of automated planning and interactive integration planning, to seek a reasonable path.
\end{abstract}

\section{Introduction}

Based on the product assembly sequence, assembly path planning design a path for the part to move from the stored position to the final assembly position. Firstly, Path planning set security as the main purpose, secondly, Path planning should meet the needs of operations and avoid the obstacles, lastly, Path planning could verify the feasibility of product design and assembly sequence planning. Assembly path can be two-dimensional can also be a three-dimensional, can be simple straight line can also be a complex multidimensional curve[1-3].

People's subjective initiative could play a large role in the interactive planning. The automated path planning in practical, such as the topological method, the moving boundary method, the space posture method, the $\mathrm{A}^{*}$ method, and so on, is difficult to automatically generate assembly path efficiently, is still in the phase of research[4,5].

In this paper, ant colony algorithm based on assembly space depth map, which could find a feasible path quickly is used for assembly path planning, at the same time could reduce searching time and cut down the path length, then make use of virtual interacting for detection and correction. $[7,8]$ Because real-time assembly path planning is really a complex engineering problem, in this article, as a study, provides a solution for assembly path planning.

\section{Automated Planning using Ant colony algorithm}

Pre-treatment.First, mesh the $3 \mathrm{~d}$ space, the assembly space coordinates $(\mathrm{x}, \mathrm{y}, \mathrm{z})$ is used to represent the basic unit of space, then set the occupied space assignment " 0 ", the rest are set to " 1 ", so generate the assembly space attribute table, which could be used to distinguish whether each coordinate assembly space is available or not.

"Man struggles upwards; Water flows downwards", water is always flowing from the high to the low, if there was a feasible path, the water would flow from starting point continuously to the finish point, so the assembly space depth attribute value could be used to represent the "elevation" of the space point. First set the depth of the assembly final point to "1", then spread the attribute value in the whole assembly space, if the nearby assembly space is available, the depth of the assembly space 
would be set to "2", in turn, set the depth of the whole assembly space attribute. As shown in figure $1(2 \mathrm{~d}$ assembly depth map) and figure ( $3 \mathrm{~d}$ assembly depth map). If there is a feasible path, then the assembly space depth value gradually rise from assembly finish point to assembly beginning point.

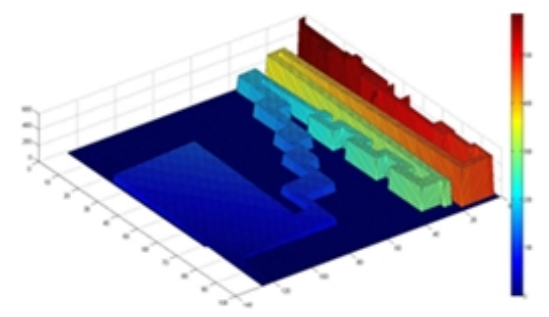

Fig. 1. 2d assembly depth map

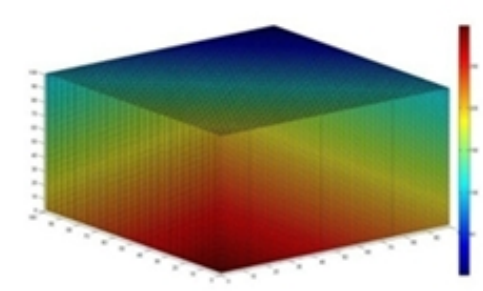

Fig. 2. 3d assembly depth map

Using space pre-treatment can not only discriminate whether there is a feasible path, but also obtain the minimum steps needed to complete the assembly path, greatly improving the efficiency of assembly path planning. In the process of solving, as to identify the assembly space, introduce the following notation:

Assembly space attribute:

$$
\mathrm{S}(\mathrm{x}, \mathrm{y}, \mathrm{z})=\left\{\begin{array}{lr}
1 & (\text { available }) \\
0 & (\text { unavailable })
\end{array}\right.
$$

Assembly space depth values:

$$
\mathrm{SD}(\mathrm{x}, \mathrm{y}, \mathrm{z})=\mathrm{i}(\mathrm{i}=1,2,3 \ldots)
$$

Automated Path Planning. The gradually reducing of assembly space depth values is the premise of the ant colony algorithm path planning, which could not only narrow the scope of the ants searching, but also limit the life of ants, in order to prevent the algorithm to a halt. Ant colony algorithm, in the process of path planning, make use of the amount of pheromone track space nodes, the depth of space, and so on, to determine the transfer direction of the next step, the total transition probability is composed of the pheromone transition probability and the distance heuristic probability.

Set $\mathrm{TP}_{(i, O r i)}^{k}(t)$ as the pheromone transition probability for the ant $\mathrm{k}$ moving from the node $\mathrm{i}(\mathrm{x}, \mathrm{y}, \mathrm{z})$ to surrounding node, at the moment $\mathrm{t}$ :

$$
\operatorname{TP}_{(i, O r i)}^{k}(t)= \begin{cases}\frac{\tau_{\text {Ori }}^{\alpha}(t) \cdot \eta_{\text {Ori }}^{\beta}}{\sum_{r \in S_{i}} \tau_{r}^{\alpha}(t) \cdot \eta_{r}^{\beta}} & \text { Ori } \in S_{i} \\ 0 & \text { else }\end{cases}
$$

Where:

$\tau_{\text {Ori }}(t) \quad$ is the Ori direction pheromone density of the node $\mathrm{i}(\mathrm{x}, \mathrm{y}, \mathrm{z})$ at the moment $\mathrm{t}$;

$\eta_{\text {ori }} \quad$ is the Local visible heuristic function, which could be standed by the reciprocal of the distance between two nodes $1 / d_{\text {Ori }}$;

$S_{i} \quad$ is the collection of the available surrounding space for ant k at the node $\mathrm{i}(\mathrm{x}, \mathrm{y}, \mathrm{z})$;

$\alpha, \beta \quad$ is the weight of $\tau_{\text {Ori }}(t)$ and $\eta_{O r i}$ in the entire transition probability.

Set $\mathrm{DP}_{(i, O r i)}(t)$ as the distance heuristic probability for the ant $\mathrm{k}$ moving from the node $\mathrm{i}(\mathrm{x}, \mathrm{y}, \mathrm{z})$ to surrounding node, at the moment $\mathrm{t}$ :

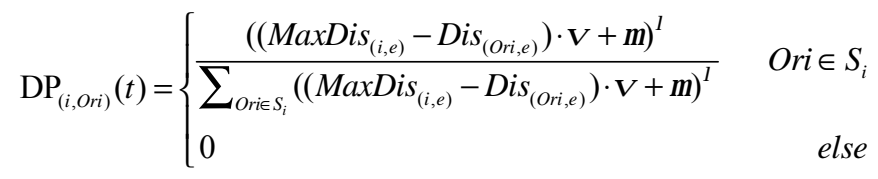

Where:

$\operatorname{Dis}_{(O r i, e)} \quad$ is distance between the node Ori and the final node e, which should be calculated before planning; 
$\operatorname{MaxDis}_{(i, e)} \quad$ is the maximum of $\operatorname{Dis}_{(\text {Ori,e) }}$;

$\varpi, \mu, \lambda \quad$ is calibration coefficient, which is used to calibrate $\operatorname{Dis}_{(\text {ori,e) }}$ in order to reflect the differences between them, prevent the distance heuristic probability becoming a random function, for the maximum difference between $\operatorname{Dis}_{(O r i, e)}$ is 2 .

So the total transition probability is:

$$
P_{(i, O r i)}^{k}(t)=\left\{\begin{array}{lr}
r \cdot T P_{(i, O r i)}^{k}(t)+(1-r) \cdot D P_{(i, O r i)} \text { if } r(\text { Ori })=S D(i)-1 \\
0 & \text { else }
\end{array}\right.
$$

Where:

$\gamma \quad$ is the proportional distribution coefficient, $\gamma \in[0,1]$, which is the weight of the pheromone transition probability and the distance heuristic probability.

To enhance the ability of global searching, a random number $q \in(0,1)$ should be generated firstly, if $q \leq q_{0}$ ( $q_{0}$ is a preset value), the total transition probability take effect, otherwise it would choose a random node among the feasible region.

$$
P_{(i, O r i)}^{k}(t)=\left\{\begin{array}{lll}
\arg \max _{u \in S_{i}}\left\{p_{(i, O r i)}\right\} & \text { if } & q \leq q_{0} \\
S_{i} & \text { if } & q>q_{0}
\end{array}\right.
$$

In order to accelerate the convergence rate and get the global optimal solution, the pheromone of each node should be update during the planning.

Whenever an ant complete the assembly path searching, the pheromone of each node on the path should be update:

$$
\tau_{i}=\rho_{\text {local }} \cdot \tau_{i}+\left(1-\rho_{\text {local }}\right) / \text { Length }
$$

Where:

$\rho_{\text {local }} \quad$ is the local residual factor of the pheromone;

Length is the length of the path.

In order to reflect the merits of merits of the different assembly paths, the evaporation rate of any node should be same, and the pheromone release is inversely proportion to the length of the path.

Whenever all ants complete the assembly path searching, the pheromone of each node on every path should be update:

$$
\tau_{i}=\rho_{\text {Glocal }} \cdot \tau_{i}+ \begin{cases}\left(1-\rho_{\text {Glocal }}\right) / \text { Length }_{\min } & \text { if } i \text { is on the best path } \\ 0 & \text { if } \quad i \text { is not on the best path }\end{cases}
$$

Where:

$\rho_{\text {Glocal }} \quad$ is the global residual factor of the pheromone;

Length $_{\text {min }} \quad$ is the length of the best path at present.

Algorithm Flow.Step 1: Read the assembly space information, preprocess the depth of assembly space attribute value.

Step 2: Set the total number of ants, and the pheromone of each node will be initialized to 0 .

Step 3: Empty the path, set the ants at starting node, and assign $\mathrm{N}$ (the depth of the starting node) to all the ants.

Step 4: Choose the next node according to the transition probability, and add the node to the path.

Step 5: Repeat steps 3 and 4, until the ant can't find a smaller value of depth or arrive at the finish node.

Step 6: Once the ant reach the finish node, record the path, then update the local pheromone.

Step 7: Repeat steps 3-6 for all the ants, and update the pheromone on the optimal path.

Step 8: Repeat step 2-7 until the result meet the conditions, then get the final output. 


\section{Interactive Planning Using Virtools}

In order to bring people's subjective initiative to full play, interactive planning is a good way to find a reasonable path. On the basis of automatic assembly path planning, make use of Virtools, which has the function of interaction and collision detection, to realize interactive planning again.

Normally, CAD software is used to build product model, firstly, the CAD model should be transformed into Virtools (*.nmo type) to carry out the following work(Fig 3). Virtools and some CAD software provide related plug-in to transform the model, in this paper, a plug-in was developed to solved the problem, Fig 3 shows how the plug-in work.

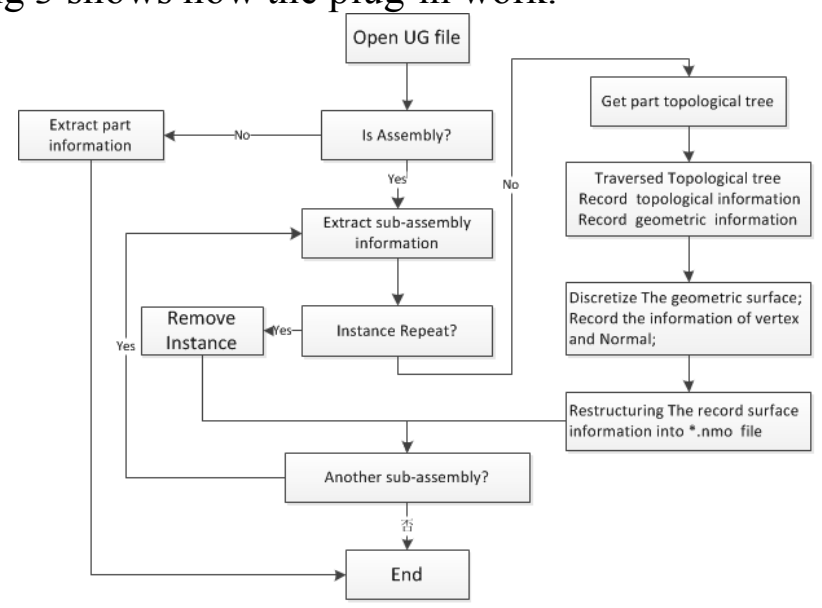

Fig 3 The flow of extracting assembly information from UG

In this paper, we take thsse check (Fig 4and Fig 5) valve as an example to carry on research.

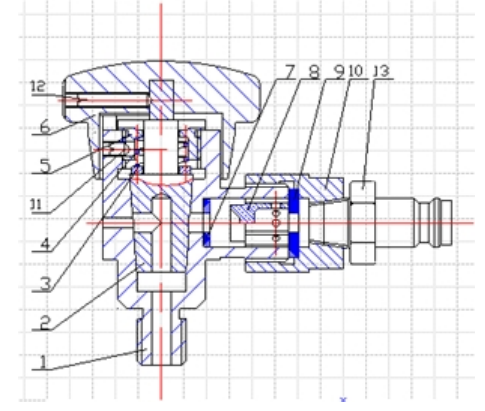

(a)

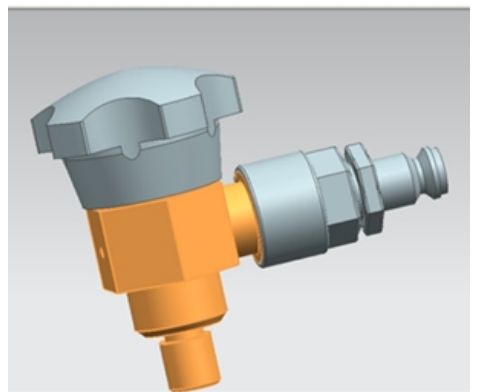

(b)

1.Valve body 2.Vlave plug 3.Gasket 4.Spring 5.Nut 6 Knob 7. Small sealing gasket 8.Slide 9.large sealing gasket 10.Joint 11 Screws $\times 4$ 12.Screws $\times 14$ 13.Pipe joint

Fig 4 The model of the check valve

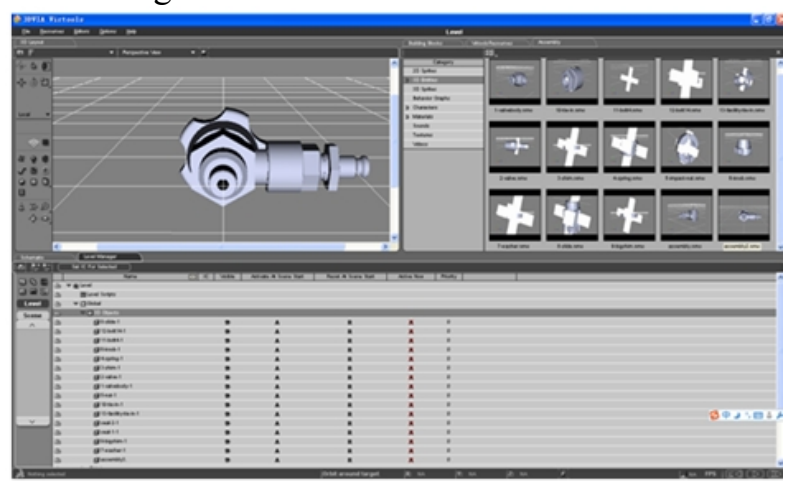

Fig. 5. The model of the check valve in Virtools

In Virtools, use the Get Position module ,Get Bounding Box module to obtain the position and bounding box of the parts, that is already assembled or not. We assume that all parts start from a 
specific point (Where the real products store).

Based on the information of VRPosAss (The position of the assembly part that are already assembled), BoxAss (The bounding box of the assembly parts that are already assembled), VRPosStart (The position of the part that should be assembled immediately), BoxPart (The bounding box of the part that should be assembled immediately), and VRPosEnd (The assembly position of the part that should be assembled immediately). In the process of planning, AABB bounding box is adopted for the assembly parts, Spherical bounding box is adopted for the unassembled part. Then the ant colony algorithm based on depth-first search could take advantage of these information to generate the corresponding assembly path, and the output path coordinates and the corresponding path curve.

If VRPosEnd is out of BoxAss, it usually would find a fit path ,for example, the large sealing gasket move from the node $(-30,-30,30)$ to the node $(25,0,0)$ (Fig 6).

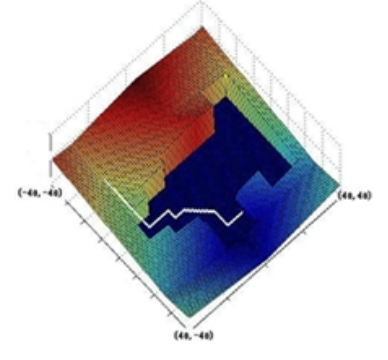

Fig 6 The path of large sealing gasket

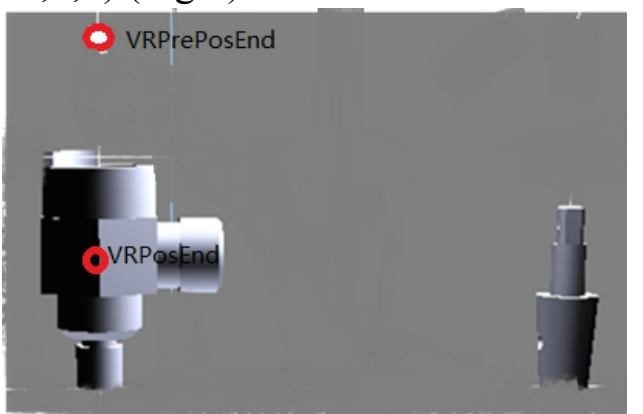

Fig 7 The node of VRPrePosEnd

In most cases, VRPosEnd isn't out of BoxAss, there is no way for the anti colony algorithm to solve the path planning problem, now introducing VRPrePosEnd (a transitional node that the unassembled part could move forward first) through interactive would be a good manner of solving the dilemma (Fig 7). Then the problem will be converted to plan a path from VRPosStart to VRPrePosEnd. For example. the valve plug move from start node to the transfer node as the first step, next the valve plug move from the transfer node to the final assembly position(Fig 8). Virtools provides Mouse Waiter module, 2D Picking module and etc for interacting with the screen and model, it means that you could interacting with the model conveniently.

Interaction provide a compromise proposal when the automatic path planning algorithm can't work intelligently, so that the problem would be solved reasonably.
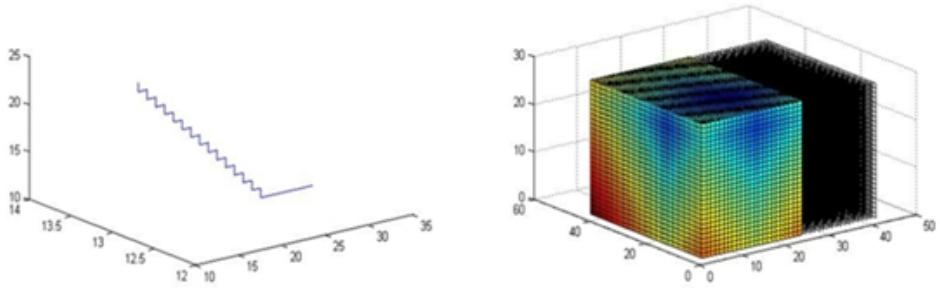

Fig 8 The path of Valve plug (Planed)

Once the path is generated, the feasible path should be verified. Utilizing the Curve Follow module, Position On Curve module, and so on, the unassembled part would move along the curve planned (Fig 9). Furthermore, there are lots of modules in Virtools ,such as Moving Obstacle module, Collision Detection module, Multi Collision Detection module, and etc, for collision detection. With function of movement and collision detection, real-time detection can realize for verifying the path (Fig10). 


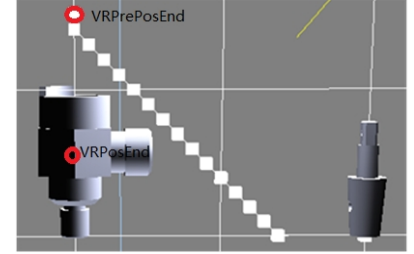

Fig 9 The path of Valve plug
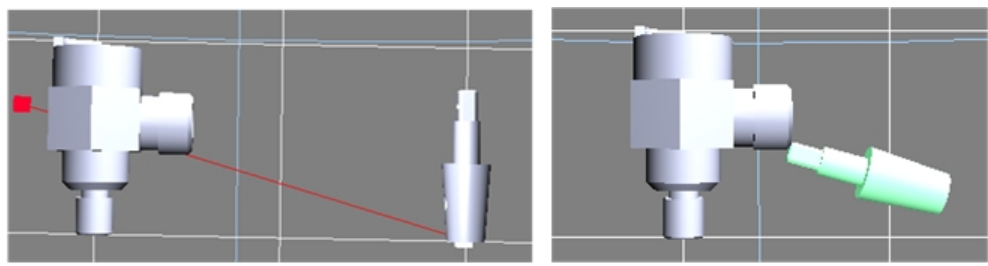

Fig 10 Collision detection Diagram

If there is interference, arrange another transitional node through interaction instead of the interference point upon the system prompts, then plan the path again until the path meet the requirements.

\section{The overview flow}

The purpose of assembly path planning is to find out a feasible path, either automatic plan or manual plan can be used to solve the problem. In this paper, automatic and manual method both play an important role. The fig 11 shows the process of this system.

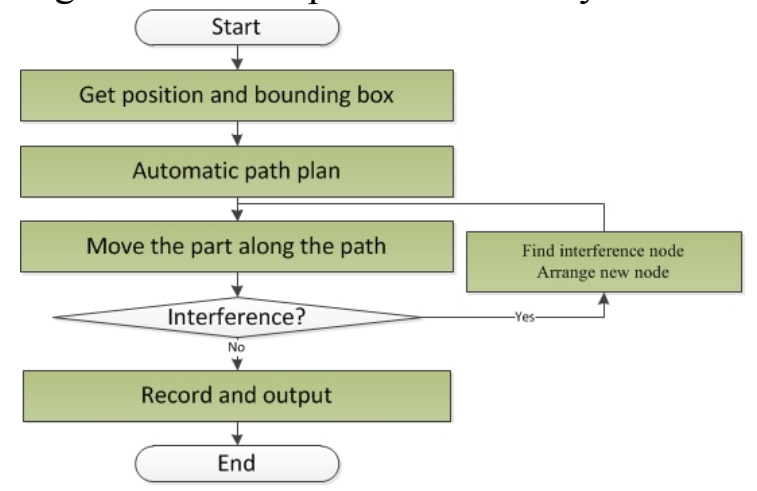

Fig 11 The process of the plan system

\section{Conclusion}

The ant colony algorithm based on depth-first search has been verified through some examples, it could find a feasible assembly path, with the help of human-interactive it would be more adaptable. Virtools provides the platform to merge manual and automatic together, bring the idea into reality.

In the following studies, detailed model, motion attitude control, kinematics parameter and so on should be considered, and smooth the path further.

\section{References}

[1] Oliver J H,Huang H T, "Automated path planning for integrated assembly design," Computer-Aided Design, Vol 26, Issue 09, pp 658-666, Sep 1994

[2] Aleksandar Shurbevski, Noriaki Hirosue, Hiroshi Nagamochi," Optimization Techniques for Robot Path Planning," Advances in Intelligent Systems and Computing, Vol 231, pp 111-120, 2014

[3] KaiWing Tang, Jarvis, R.A, "A fast algorithm to plan a collision-free path in cluttered 2D environments," IEEE Conference on Robotics, Automation and Mechatronics, 2004 IEEE Conference on, Vol 2, pp 786-791, Dec 2001

[4] LIU Jian-hua, NING Ru-xin, WAN Bi-le, XIONG Zhen-qi, "Research of Complex Product Assembly Path Planning in Virtual Assembly," Journal Of System Simulation, Vol 19, Issue 09, Jun 2007

[5] Liangjun Zhang, Xin Huang, Young J. Kim, Dinesh Manocha,'D-Plan: Efficient Collision-Free Path Computation For Part Removal and Disassembly," Journal of Computer-Aided Design and Applications, Vol 5, Issue 6, pp 774-786, 2008 
[6] Zeyuan Lu, Tijing Cai, Zhuopeng Yang, "Path Plan in Gravity Aided Inertial Navigation Based on Ant Colony Algorithm," IEEE Conference: Intelligent Systems, Vol 1, pp 193-198, May 2009

[7] Cheng Hui, Li Yuan, Zhang Kai-fu, " Efficient method of assembly sequence planning based on GAAA and optimizing by assembly path feedback for complex product," The International Journal of Advanced Manufacturing Technology, Vol 42, Issue 11-12, pp 1187-1204, Jun 2009 